1 Running head: Flower microbiome assembly in almond

3 Title: Ecological dynamics of the almond floral microbiome in relation to crop management and

4 pollination

6 Authors: Robert N. Schaeffer ${ }^{1,2,5}$, David W. Crowder ${ }^{1}$, Javier Gutiérrez Illán ${ }^{1}$, John J. Beck ${ }^{3}$,

7 Tadashi Fukami ${ }^{4}$, Neal M. Williams ${ }^{2}$, and Rachel L. Vannette ${ }^{2}$

9 Author affiliations:

$10 \quad{ }^{1}$ Department of Entomology, Washington State University, Pullman, WA 99164

$11{ }^{2}$ Department of Entomology \& Nematology, University of California Davis, Davis, CA 95616

$12{ }^{3}$ Chemistry Research Unit, Center for Medical, Agricultural, and Veterinary Entomology,

13 Agricultural Research Service, United States Department of Agriculture, Gainesville, FL 32608

$14{ }^{4}$ Department of Biology, Stanford University, Stanford, CA 94305

\title{
$16{ }^{5}$ Corresponding author and current address:
}

17 Robert N. Schaeffer

18 Department of Biology

19 Utah State University

20 Logan, UT 84322

21 E-mail: robert.schaeffer@usu.edu 


\section{Abstract}

23 1. Crop tissues harbor microbiomes that can affect host health and yield. However, processes driving microbiome assembly, and resulting effects on ecosystem services, remain poorly understood. This is particularly true of flowering crops that rely on pollinators for yield.

2. We assessed effects of orchard management tactics and landscape context on the flower microbiome in almond, Prunus dulcis. Fourteen orchards (5 conventional, 4 organic, 5 habitat augmentation) were sampled at two bloom stages to characterize bacterial and fungal communities associated with floral tissues. The surveys were complemented by in vitro experiments to assess effects of arrival order and fungicides on nectar microbial communities, and effects of fungicides and microbes on honey bee foraging. Finally, a field trial was conducted to test effects of fungicides and microbes on pollination.

3. As bloom progressed, bacterial and fungal abundance and diversity increased, across all floral tissue types and management strategies. The magnitude by which microbial abundance and diversity were affected varied, with host proximity to apiaries and orchard management having notable effects on bacteria and fungi, respectively. reduced nectar removal. Neither treatment affected pollination services. practices can shape microbiota associated with flowers of a pollinator-dependent crop. With growing appreciation for the role of floral-associated microbes in affecting biotic interactions at the floral interface, understanding such drivers can potentially inform 
microbial-derived ecosystem services in agricultural landscapes, including pollination and biocontrol.

47 Keywords: agricultural landscapes, ecosystem services, flower microbiome, fungicide,

48 pollination, Prunus dulcis, sustainable agriculture

Introduction

Crop tissues harbor distinct microbiomes that affect host health and yield. For example,

52 microbiomes can affect host tolerance to stress and disease (Vurukonda et al., 2016; Berg and

53 Koskella, 2018) and can stimulate growth through mobilization and transport of nutrients (Pii et

$54 a l ., 2015)$. However, despite the importance of the microbiome, few studies have investigated the

55 processes mediating microbiome assembly, especially in crop systems (but see Edwards et al.,

56 2015; Grady et al., 2019). Assessing such processes is critical, as manipulation of the plant

57 microbiome can have wide-ranging effects, including improved crop performance, food safety,

58 associated ecosystem services, and sustainability (Mueller and Sachs, 2015; Busby et al., 2017;

59 Toju et al., 2018; Allard and Micallef, 2019).

60 Approximately $35 \%$ of crops produced globally benefit from pollination by arthropods

61 (Klein et al., 2007). With an economic value exceeding $\$ 300$ billion globally (Lautenbach et al.,

62 2012), there is strong incentive to manage pollination of flowering crops during bloom. Bloom

63 management is also critical because some plant pathogens infect crops through flowers and can

64 be dispersed by pollinators (McArt et al., 2014). To combat pathogens, growers often use

65 fungicides, bactericides, or other antibiotics during bloom. However, such chemicals can cause

66 non-target effects on the microbiome (Schaeffer et al., 2017) and affect pollinators (Frazier et al.,

67 2015; Johnson, 2015). 
In addition, agricultural intensification often decreases native pollinator abundance and diversity, largely through habitat loss (Kremen et al., 2002; Kovács $\square$ Hostyánszki et al., 2017;

Grab et al., 2019). Given that pollinators harbor distinct microbiomes and disperse microbes

71 between flowers, loss of pollinator biodiversity and reduced pollinator visitation may indirectly

72 affect microbiome assembly (Vannette and Fukami, 2017; Russell et al., 2019). In orchard

73 systems, flowering strips, hedgerows, and nesting structures have been employed to support

74 pollinator populations (Scheper et al., 2015; Williams et al., 2015; Kremen et al., 2019).

75 However, whether restored vegetation, which can act as a source of inocula, affects the assembly

76 of microbiomes in crop systems remains poorly understood (Lindow and Andersen, 1996;

77 Lymperopoulou et al., 2016).

Here, we assessed how agricultural management affected flower microbiome assembly and function in mass-flowering almond (Prunus dulcis). Almond depends on biotic pollination for

80 fruit set, and is heavily managed during bloom to ensure adequate pollination and prevention of

81 pathogens, namely Monilinia laxa, the causal agent of brown rot blossom blight. Synthetic

82 fungicides are applied during bloom to preempt $M$. laxa establishment in conventional orchards

83 (Adaskaveg et al., 2017). However, increasing demand for sustainably produced almonds has

84 spurred adoption of organic management tactics in many orchards (Brodt et al., 2009; Plattner et

85 al., 2013), including use of copper and other materials instead of synthetics for disease control.

86 Given that pollinators of almond, including honey bees and bumble bees, are sensitive to the

87 chemical alterations that nectar microbes induce through metabolism of nectar resources (Rering

88 et al., 2018; Schaeffer et al., 2019), shifts in microbiome structure arising from different

89 management schemes may affect pollination services (Herrera et al., 2013; Vannette et al., 2013;

90 Schaeffer and Irwin, 2014). 
To address these linkages, we conducted a field survey of microbial diversity on almond

92 flowers in orchards with different management schemes. We also conducted two experiments to

93 examine mechanisms that affect microbial assembly and function in almond. First, we examined

94 how fungicides interact with variation in microbial immigration history to affect microbial

95 community structure. Second, we examined effects of nectar microbes and fungicides on honey

96 bee foraging, and consequences for pollination. Overall, our results provide evidence that

97 variation in management of a blooming crop can shape microbiome assembly and the biotic

98 interactions that mediate ecosystem services in agricultural landscapes.

\section{Methods}

\section{$101 \quad$ Study system}

California (CA) almonds flower in February through early March, and rely almost

103 exclusively on managed Apis mellifera colonies for pollination. Approximately 470,000 ha of

104 almond orchards in CA produced over 80\% of the world's supply in 2018, generating \$5.8B in

105 farmer revenue (Sumner et al., 2014; Almond Board of California, 2019). Despite these

106 numbers, fruit set in almond orchards typically ranges from 10-40\% (Bosch and Blas, 1994),

107 partly due to limited pollinator availability and the quality of services they provide, in addition to

108 disease and inclement weather during bloom.

109

\section{Orchard survey}

111 We surveyed 14 orchards (4 organic, 5 conventional, and 5 with supplemental forb

112 plantings) across the Sacramento Valley of CA (Figure 1). Supplemental forb plantings included

113 a mix of annual species native to CA, including Calandrinia ciliata, Collinsia 


\section{4 heterophylla, Eschscholzia californica, Nemophila maculata, Nemophila menziesii, Phacelia}

115 campanularia, and Phacelia ciliata. Beyond the addition of forb plantings, forb-amended

116 orchards follow conventional management practices. Orchards were sampled twice between

117 February 15 and 24, 2017, once at early bloom ( 10\% of flowers open) and then at peak bloom

118 (>50\% of flowers open). At each orchard and sampling event, six trees ('Nonpareil' variety)

119 were sampled; three near the edge of the orchard, and adjacent to the forb planting if available.

120 These trees were located in the second row in from the edge of the orchard ('edge'). The other

121 three trees were sampled from the orchard interior (row 10) ('interior'). We chose this sampling

122 scheme because semi-natural habitat in the surrounding landscape can increase visitation by

123 native pollinators such as bees and flies (Klein et al., 2012). Pollinators can also be important

124 dispersal agents for microbes (Aizenberg-Gershtein et al., 2013; Vannette and Fukami, 2017);

125 thus, we may see greater microbial abundance or diversity in flowers in close proximity to these

126 natural habitats. For each site (edge or interior) and sampling event, 30 open flowers $(N=10$ per

127 tree) were collected using aseptic technique and then pooled at the site level. Flowers with flat,

128 fully-reflexed petals that had been open for approximately 3 days were chosen for collection (Yi

129 et al., 2006). This choice increased the probability that flowers had been visited by pollinators

130 that disperse microbes. Once collected, flowers were placed in a cooler and transferred to the lab,

131 then stored at $4{ }^{\circ} \mathrm{C}$ until processing (within the following $24 \mathrm{~h}$ ).

132 To assess effects of pollinator foraging activity on microbiome structure and diversity, we

133 used two complementary approaches. First, during flower collection, we measured the distance

134 between trees sampled and the nearest set of honeybee hives in the orchard. Honey bees often

135 forage near their hive (Gary et al., 1978), and we hypothesized that tree proximity would be a

136 proxy for visitation frequency during bloom. Second, we measured the amount of semi-natural 
137 habitat within $1 \mathrm{~km}$ buffer of each orchard, as prior work shows increased semi-natural habitat

138 can increase the diversity and visitation rates of native pollinators in orchards (Klein et al.,

139 2012). We classified land cover from the croplands data layer product (USDA, 2017) within a 1-

$140 \mathrm{~km}$ radius of each orchard edge using ArcGIS (ESRI, Redlands, CA, USA). Natural habitat near

141 orchards in this study can primarily be classified as chaparral, oak woodland, or valley and

142 foothill riparian woodland (Barbour et al., 2007). We predicted that more natural habitat would

143 promote more diverse floral microbiomes as pollinator species harbor distinct microbiomes

144 (Koch et al., 2013; Graystock et al., 2017) and disperse microbes (Vannette and Fukami, 2017;

145 Russell et al., 2019).

\section{Sample processing}

148 Whole flowers were dissected acropetally in sequence to minimize cross-contamination, as

149 previous work has shown that floral microbial communities can display taxonomic-structuring

150 across tissue types (Junker and Keller, 2015). Petals were removed first using sterile forceps.

151 Petals from all 30 flowers in a sample were then pooled in a $50 \mathrm{~mL}$ Falcon tube (Corning,

152 Corning, NY, USA), massed for fresh weight (g), then suspended in $20 \mathrm{~mL}$ of $1 \mathrm{x}-0.15 \%$ PBS-

153 Tween solution. The androecium and gynoecium (hereafter collectively referred to as 'Anthers')

154 were then removed from the base of each flower, pooled in a $15 \mathrm{~mL}$ Falcon tube, massed, then

155 suspended in $5 \mathrm{~mL}$ of $1 \mathrm{x}-0.15 \%$ PBS-Tween solution. To sample nectar, we 'washed' each

156 hypanthia with $2 \mu \mathrm{L}$ of $1 \mathrm{x}-0.15 \%$ PBS-Tween solution using a pipette, and pooled for each set of

15730 flowers. Each wash was diluted with $1 \mathrm{~mL}$ of $1 \mathrm{x}-0.15 \%$ PBS-Tween solution. For petals,

158 androecium, and gynoecium, samples were sonicated (Branson CPX5800H, Danbury CT) for 10

159 min to dislodge epiphytic microbes. After sonication, debris was removed from sample tubes by 
160 pouring samples through autoclaved cheesecloth into a sterile Falcon tube. Falcon tubes

161 containing debris-filtered samples were then centrifuged at $3000 \mathrm{rpm}$ for $10 \mathrm{~min}$ at $4{ }^{\circ} \mathrm{C}$ to pellet

162 microbial cells. We then decanted the supernatant, re-suspended microbial cell pellets in $1 \mathrm{~mL}$ of

163 autoclaved PBS solution, vortexed tubes, then transferred the cell suspensions to new $1.7 \mathrm{~mL}$

164 microcentrifuge tubes.

165

Microbial abundance

To estimate microbial abundance across tissue types, we used dilution plating to estimate

168 the density of colony forming units (CFUs) for each sample. Selective media for growth of fungi

169 (yeast malt agar + chloramphenicol) and bacteria (R2A + cycloheximide) was used. Although

170 not all microbes are culturable, previous work suggests that most dominant species observed to

171 be associated with flowers are culturable on these media types (Morris et al., 2020). Plates were

172 incubated for 5 days at $25^{\circ} \mathrm{C}$ and colonies counted.

173

174 DNA extraction and sequencing

175 Genomic DNA was extracted from samples using a ZymoBIOMICS® DNA Microprep kit

176 (Zymo Research, Irvine, CA, USA) at the University of California, Davis (Davis, CA, USA),

177 following the manufacturer's protocol. Extracted DNA was then sent to the Centre for

178 Comparative Genomics and Evolutionary Bioinformatics at Dalhousie University (Halifax, Nova

179 Scotia, Canada) for library preparation and 16S/ITS amplicon sequencing. Amplicon sequence

180 variants (ASVs) were assigned using DADA2 (Callahan et al., 2016). See SI Materials and

181 Methods for information concerning amplification, sequencing, and bioinformatic processing of

182 data. 


\section{Microbial community assembly}

To assess effects of nectar composition, two different fungicides, and arrival order on microbiome assembly, we conducted a sequential inoculation experiment. We used three

187 different synthetic nectar environments containing either copper, typically used in organic

188 orchards, or propiconazole, a synthetic, demethylation inhibitor (DMI) often used in

189 conventional orchards. We used microbes commonly observed in nectar, including Asaia astilbis

190 (bacterium), Aureobasidium pullulans (yeast), Metschnikowia reukaufii (yeast),

191 and Neokomagataea thailandica (bacterium). These species are frequently found on floral tissues

192 and nectar, including almond (Fridman et al., 2012; Aizenberg-Gershtein et al., 2013; Schaeffer

193 et al., 2017). Colonies formed by these species are distinguishable on media. Strains used were

194 isolated from almond nectar or Epilobium canum (Onagraceae), a perennial herb native to the

195 foothills of California (Morris et al., 2020). Yeast and bacteria strains were cultured on YMA

196 and R2A, respectively, and grown at $25^{\circ} \mathrm{C}$. After three days of growth, microbial cell

197 suspensions for inoculation were diluted to ca. 400 cells $\mu \mathrm{L}^{-1}$ using a hemocytometer just prior

198 to the beginning of the experiment described below.

199 To prepare the synthetic nectar environments, fungicides were added to filter-sterilized

$20015 \%(\mathrm{w} / \mathrm{v})$ glucose:fructose solution supplemented with $0.32 \mathrm{mM}$ amino acids from digested

201 casein (Vannette \& Fukami 2014). Fungicide concentration (7500 ppb) was parameterized based

202 on residue analyses of almond flowers and those of other flowering crops (Frazier et al., 2015).

203 In each nectar environment, we assessed the strength of priority effects following a two-way,

204 full-factorial design, with three different orders of species introductions (e.g., Tucker and Fukami

205 2014). Our introduction treatment groups included: (i) simultaneous introductions of the two 
206 yeast and bacterial species to the artificial nectar on day 0; (ii) 'yeast-first' sequential

207 introductions, in which the two yeast species were introduced first (day 0) and then, $48 \mathrm{~h}$ later,

208 the two bacterial species; and (iii) 'bacteria-first' sequential introductions, in which the two

209 bacterial species were introduced first (day 0) and then, $48 \mathrm{~h}$ later, the two yeast species. The

210 experiment was performed in $200 \mu \mathrm{L}$ polymerase chain reaction (PCR) tubes (ThermoFisher

211 Scientific Corp.) and lasted for $4 \mathrm{~d}$, which approximates the lifespan of an almond flower (Yi et

212 al., 2006). To each tube, we added $9 \mu \mathrm{L}$ of synthetic nectar at the start, and $0.5 \mu \mathrm{L}$ suspensions of

213 each respective microbial species (ca. 200 cells) for the appropriate day and treatment

214 combination. We chose this timing as $48 \mathrm{~h}$ as a realistic time interval between microbial

215 immigration events in floral systems with displays open for such a duration (Peay et al., 2012;

216 Vannette and Fukami 2014). Four days after the first inoculation the experiment was ended and

217 nectar from each tube was divided for chemical analysis and to determine microbial abundance.

218 All treatment combinations were performed in each of the three nectar environments $(N=16$ per

219 treatment combination).

\section{Pollination}

222 Pollination Experiment One - To test consequences, including non-additive effects, of

223 flower exposure to fungicides and microbes on pollinator foraging behavior, we performed a

224 field assay. Briefly, artificial flowers (Figure S2) designed to mimic those of almond were set out

225 in an array near an apiary at the Harry H. Laidlaw Jr. Honey Bee Research Facility (Davis, CA).

226 Flowers were treated with $200 \mu \mathrm{L}$ of artificial nectar (same as above) in a fully-crossed design,

227 with three levels for each treatment. For fungicides, treatment levels were: (1) no fungicide

228 (control), (2) organic (copper, $7500 \mathrm{ppb),} \mathrm{or} \mathrm{(3)} \mathrm{conventional} \mathrm{(propiconazole,} 7500 \mathrm{ppb).} \mathrm{With}$ 
respect to nectar-inhabiting microbes, treatments were: (1) no bacterium or yeast (control), (2) $N$.

230 thailandica (bacterium), or (3) M. reukaufii (yeast). Experimental arrays were set $\sim 1-2 \mathrm{~m}$ from

231 the hives at the apiary in the morning each day the experiment was performed. Two hours after

232 the start of the experiment each day, the remaining nectar from each flower's tube was capped,

233 brought back to the laboratory, and weighed to estimate changes in volume. This assay was

234 performed four times ( $N=12$ flowers per treatment combination per assay). See SI Materials and

235 Methods for full experimental details.

236 Pollination Experiment Two - We performed an in vivo field assay at an orchard (Zamora,

237 CA, USA) to test for consequences, including non-additive effects, of flower exposure to

238 fungicides and microbes on the quality of pollination services. Briefly, fungicide/microbe

239 treatments mirrored those used in the first pollination assay, with treatment identity randomized

240 among 9 unvisited flowers within an individual tree $(N=20$ 'Nonpareil' variety trees, spaced

241 across alternating rows, with five haphazardly selected in each row). Two microliters of treated

242 nectar was applied to each flower, and after two days of exposure to pollinators, flowers were

243 carefully removed along with the pedicel and placed in individual $1.5 \mathrm{~mL}$ microcentrifuge tubes

244 containing $0.5 \mathrm{~mL}$ of water. Flowers were positioned such that the stigma did not touch the

245 tube's surface and the pedicle was in water (Brittain et al., 2013). Once returned to the lab,

246 flowers were stored in the dark at room temperature for $72 \mathrm{~h}$ to allow pollen tube growth. After

247 this period, pistils were fixed (Farmer's fixative) and then stored at $4{ }^{\circ} \mathrm{C}$ until further processing.

248 Pollen tube growth was assessed using a staining and microscopy procedure, following a

249 previously established protocol (Brittain et al., 2013). See SI Materials and Methods for full

250 experimental details. 


\section{Statistical analyses}

All analyses were performed in R v.4.0.2 (R Core Team, 2013). We fit linear mixed-effect

254 models with the $l m e 4$ package (Bates et al., 2014) to assess the impact of orchard management,

255 and other measured variables on microbial abundance (log-10 transformed CFU counts) and

256 diversity (ASV richness and Shannon diversity index). For each model and response variable

257 examined, management, bloom stage (early/peak), site (edge/interior) and a three-way

258 interaction among them, along with amount of semi-natural habitat surrounding orchards and

259 distance to the nearest apiary were included as predictors, with orchard identity as a random

260 effect to account for repeated sampling. Bacterial and fungal data were analyzed separately for

261 each floral tissue examined. Once fit, we used backward stepwise model selection in the

262 ImerTest package (Kuznetsova et al., 2017) to identify the best-fit model for each response

263 variable examined. Fixed model terms were retained based on log-likelihood ratio tests, with

264 significance of each calculated using $F$ tests, based on Satterthwaite approximation for

265 denominator degrees of freedom (Kuznetsova et al., 2016). Finally, to determine if the relative

266 abundance of individual ASVs responded to orchard variables of interest, we used DESeq2 with

267 Benjamini-Hochberg corrections for multiple testing (Love et al. 2014). Orchard management,

268 bloom stage, and apiary distance were treated as predictors in separate models.

269 Pairwise dissimilarities between fungal and bacteria communities were calculated using

270 the Bray-Curtis dissimilarity metric. We also calculated abundance-weighted UniFrac distances

271 for bacteria; the UniFrac metric uses phylogenetic information to calculate dissimilarities

272 between communities, and was weighted by the relative abundance of ASVs within a sample

273 (Lozupone and Knight, 2005). Finally, we used permutational multivariate analysis of variance

274 (PERMANOVA) to assess the contribution of management, bloom stage (early/peak), site 
275 (edge/interior) and all (2- and 3-way) interactions among them, along with amount of semi-

276 natural habitat surrounding orchards and distance to the nearest apiary on community

277 composition. This analysis was performed using vegan, based on 1000 permutations (Oksanen et

278 al., 2015).

To assess effects of fungicide identity and immigration order on microbiome assembly in

280 our in-vitro experiment, we fit linear mixed-effect models for each species with abundance

281 (CFUs) as the response variable and immigration order and fungicide treatment as fixed factors,

282 including their interaction.

283 For the behavior assay (Pollination Experiment One), we fit a linear mixed-effects model

284 with nectar remaining as the response, fungicide and microbe treatments as fixed factors, as well

285 as their interaction. Trial number was included as a random effect. For the in-orchard pollination

286 service assay (Pollination Experiment Two), we also fit linear mixed-effects models with pollen

287 germination and number of tubes as response variables, fungicide and microbe treatments as

288 fixed factors, as well as their interaction. Tree identity was included as a random effect.

290 Results

291 Microbial abundance and diversity

292 Floral microbial abundance and diversity increased from early to peak bloom across all

293 tissue types, and management strategies, for both bacteria and fungi (Tables S1-S3). The

294 magnitude in which microbial abundance increased, however, varied considerably among these

295 factors. Culturable bacterial CFU abundance from petals $\left(F_{1,53}=645.35, P<0.0001\right)$, anthers

$296\left(F_{1,43.92}=466.26, P<0.0001\right)$, and nectaries $\left(F_{1,54}=929.91, P<0.0001\right)$ was eleven-, five- , and

297 eight-fold higher at peak bloom, respectively (Figure 2; Table S1) than at the initial sampling. 
Like bacteria, fungal CFU abundance (Table S1) also increased from early to peak bloom, increasing two-fold for petals $\left(F_{1,46.03}=6.38, P=0.02\right)$, two-fold for anthers $\left(F_{1,52}=70.97, P<\right.$ $0.0001)$, and three-fold for nectaries $\left(F_{1,44.04}=41.11, P<0.0001\right)$. $27 \%$ more abundant in organic orchards than those that were forb-amended or conventional in management practice $\left(F_{2,52}=4.61, P=0.01\right)$. Moreover, regardless of management scheme,

305 fungi associated with petals were $11 \%$ more abundant along the edges of orchards compared to

306 the interior $\left(F_{1,46.03}=6.38, P=0.02\right)$. However, no effect of sampling location within orchards

307 was detected for bacteria, nor for the amount of natural habitat in the surrounding landscape on 308 the abundance of either bacteria or fungi.

310 (richness: $F_{1,54}=8.85, P<0.01$; Shannon index: $F_{1,54}=6.05, P=0.02$ ), anthers (richness:

$311 F_{1,35.60}=25.99, P<0.0001$; Shannon index: $F_{1,51}=33.28, P<0.0001$ ), and nectaries (richness:

$312 \quad F_{1,40.32}=102.96, P<0.0001$; Shannon index: $F_{1,40.17}=25.45, P<0.001$ ) (Figure 3; Tables S2

313 and S3). All tissues were dominated by Proteobacteria (Figure 4), particularly members of the

314 Pseudomonadaceae, with two taxa being enriched from early to peak bloom overall (BactSeq2:

315 Pseudomonas sp., log2-fold change $=2.50, P_{a d j}<0.001$; BactSeq10: Pseudomonas sp., $\log 2$-fold

316 change $\left.=3.64, P_{a d j}<0.01\right)$. Tree proximity to apiaries within an orchard was found to be

317 associated with bacterial diversity, although the association was weak (Figure S2A): Shannon

318 diversity was higher on flowers closer to apiaries than those that were collected further away,

319 though a significant effect was only detected for anthers $\left(F_{1,51}=7.39, P<0.0001\right)$ in our models. 
With the exception of organic orchards, fungal ASV richness generally increased over

321 bloom (Figure 3). This trend was significant for nectaries (richness: $F_{1,42.37}=6.81, P=0.01$ ) and

322 petals (richness: $F_{1,41}=5.32, P=0.03$ ). Shannon diversity followed a similar pattern, with a

323 significant increase observed for nectaries $\left(F_{1,42.44}=10.22, P<0.01\right)$ and petals; however, for the

324 latter this effect depended on orchard management scheme (Management $\times$ Bloom stage: $F_{2,39}=$

$3253.50, P=0.04)$. Specifically, fungal diversity (Shannon index) increased in both conventional

326 and forb-amended orchards by $59 \%$ and $12 \%$ respectively, while decreasing in organic orchards

327 by $26 \%$. Other orchard-level variables examined, including apiary distance, natural habitat in the

328 surrounding landscape, and site sampled within orchards, had no effect on observed fungal ASV

329 richness or Shannon diversity (Tables S2 and S3). We also detected no correlation between

330 apiary distance and either diversity metric (Figure S2b); however, DESeq2 analyses revealed

331 Vishniacozyma carnescens (syn. Cryptococcus carnescens) ASVs that significantly declined in

332 abundance with tree distance from apiaries (FunSeq13: $\log 2$-fold change $=-0.02, P_{a d j}<0.001$;

333 FunSeq58: $\log 2$-fold change $\left.=-0.02, P_{a d j}<0.01\right)$.

$334 \quad$ Fungal communities associated with floral tissues were generally dominated by members

335 of the Aureobasidiaceae and Bulleribasidiaceae (Figure 4), including Aureobasidium pullulans,

336 V. victoriae (syn. C. victoriae), and V. carnescens. Over bloom, A. pullulans in particular was

337 found to significantly increase in relative abundance (FunSeq1: $\log 2$-fold change $=2.96, P_{a d j}<$

338 0.0001), along with Gelidatrema spencermartinsiae (syn. C. spencermartinsiae, FunSeq16: log2-

339 fold change $\left.=2.27, P_{a d j}<0.01\right)$, Filobasidium wieringae $($ syn. $C$. wieringae, FunSeq34: $\log 2-$

340 fold change $=3.87, P_{a d j}<0.0001$ ), and Buckleyzyma aurantiaca (syn. Rhodotorula aurantiaca,

341 FunSeq26: $\log 2$-fold change $\left.=1.14, P_{a d j}<0.01\right)$. Taxa observed to significantly decline in

342 relative abundance included Cladosporium delicatulum (FunSeq3: $\log 2$-fold change $=-1.52, P_{\text {adj }}$ 
$343<0.001$ ), a widely distributed saprobe species, and Naganishia friedmannii (syn. C. friedmannii;

344 FunSeq27: $\log 2$-fold change $\left.=-3.28, P_{a d j}=0.02\right)$.

345 Bacterial and fungal species composition differed between sampling times (Table 1), with

346 bloom stage explaining 3-21\% of variation in composition, depending on flower tissue. Orchard-

347 level predictors (e.g., natural habitat) generally explained less variation in composition (Table 1).

348 Orchard management was not associated with bacterial species composition, but did predict

349 variation in fungal composition in anthers $\left(R^{2}=0.073, F_{2,33}=2.11, P=0.001\right)$ and petals $\left(R^{2}=\right.$

$\left.3500.078, F_{2,33}=2.60, P=0.005\right)$. The amount of semi-natural habitat in the surrounding landscape,

351 as well as apiary distance, were both generally found to be associated with shifts in bacterial and

352 fungal community composition, with a particularly notable effect of apiary distance on bacteria

353 in nectar $\left(R^{2}=0.176, F_{2,20}=5.65, P=0.001\right)$.

\section{Microbial community assembly in floral nectar}

356 In the in-vitro sequential inoculation experiment, arrival order significantly affected

357 species' densities in nectar. However, this effect depended on the nectar environment and

358 presence of fungicide residues (Figure 5). The bacterium Asaia was the only species that could

359 persist across all nectar environments $\left(F_{2,134}=2.64, P=0.08\right)$. Interestingly, the bacterium Asaia

360 reached higher densities when introduced after or simultaneously with yeast species $\left(F_{2,134}=\right.$

$36122.89, P<0.0001)$. Of the yeasts, persistence and growth were strongly dependent on the nectar

362 environment: Metschnikowia grew best in control nectar $\left(F_{2,134}=31.63, P<0.0001\right)$, while

363 Aureobasidium performed better in the copper treatment $\left(F_{2,134}=16.94, P<0.0001\right)$. Finally,

364 propiconazole had strong inhibitory effects on the growth of both species. 


\section{Microbial and fungicide effects on honey bee foraging and pollination}

Fungicides reduced the amount of nectar removed by honey bee foragers $\left(F_{2,311}=130.76\right.$, $P<0.0001)$. In contrast, neither microbe affected forager nectar removal $\left(F_{2,311}=1.90, P=\right.$ 0.15), nor did we detect a significant interaction between microbial inoculation and fungicide treatment $\left(F_{4,311}=2.19, P=0.07\right)$. In the orchard experiment, neither fungicide application nor nectar-inhabiting microbes affected pollen germination or pollen tube number (Table S5).

\section{Discussion}

The results presented here demonstrate that orchard management practices can mediate flower microbiome structure, though the magnitude of these effects can hinge on a variety of factors. Of those examined, timing of bloom was the most consistent predictor: bacterial and

377 fungal abundance and diversity were higher at peak bloom than at the start across all floral tissue

378 types and management strategies. Sampling time with respect to bloom intensity has been

379 documented previously (e.g., Smessaert et al., 2019), and may be driven by variation in a

380 combination of interrelated variables, including temperature, pollinator activity, and host

381 metabolism. However, other orchard-level variables affected microbial abundance and diversity,

382 with host proximity to apiaries and orchard management having significant effects on both

383 bacteria and fungi, respectively.

The results described here support a key role for pollinator foraging in the assembly of the

385 almond flower microbiome. For example, the orchard survey detected a strong signature of tree

386 proximity to apiaries on epiphytic bacterial diversity of almond flower nectaries and reproductive

387 structures, as both bacterial richness and diversity decreased the further trees were from apiaries.

388 Within orchards, honey bees forage more intensively near their apiary (Bates et al., 2014), 
dispersing bacteria among flowers (Vannette and Fukami, 2018; Russell et al., 2019), and generate spatial variation in microbial transmission and resulting flower microbial communities.

391 Moreover, while foraging on flowers, individual honey bees can display consistent behaviors,

392 including focus on either pollen and nectar collection only, affecting the degree of contact with

393 intrafloral tissues (Bosch and Blas, 1994; Thomson and Goodell, 2001). Finally, although contact

394 with petals can occur as foragers side-work for nectar (Thomson and Goodell, 2001), these

395 collective behaviors may explain the lack of a significant effect of apiary distance on bacterial

396 diversity observed on petals vs. nectar and reproductive structures of the flower. In sum, these

397 patterns point to increased consideration of both apiary spacing, and pollinator foraging

398 behavior, as interest grows in leveraging pollinators as vectors of microbial biocontrol agents to

399 combat disease (Kevan et al., 2008; Menzler-Hokkanen and Hokkanen, 2017).

$400 \quad$ Pollinator foraging could also affect microbial arrival to flowers and community assembly,

401 generating priority effects between microbial species (Peay et al., 2011; Vannette and Fukami,

402 2014). Indeed, in our laboratory experiment, immigration history had a significant effect on

403 species' persistence and densities in nectar. These effects, however, were dependent on both the

404 biotic environment and presence of fungicides. At the concentrations tested, both fungi were

405 highly susceptible to the synthetic fungicide propiconazole, while Aureobasidium was much less

406 so in comparison to Metschnikowia when challenged with copper. Surprisingly, the bacterium

407 Asaia persisted in all nectar environments, while Neokomagataea did not, in contrast to previous

408 findings (Tucker and Fukami, 2014). Additional work on the sensitivity of these taxa to

409 fungicides across different sugar environments may explain these patterns. Taken together, these

410 results suggest that arrival order and environmental filtering via the presence of fungicides can

411 potentially affect beta diversity among nectar microbial communities in managed flowering 
412 systems. As nectar-inhabiting fungi and bacteria can differ in their effect on nectar chemistry

413 (Vannette and Fukami, 2018), it is possible that microbial effects stemming from differing

414 community compositions may manifest to affect the quality of pollination services provided

415 among managed flowering systems.

416 Honey bee foraging was affected by fungicides, but not by nectar-inhabiting microbes, in

417 contrast to previous work (Vannette et al., 2013; Good et al., 2014; Rering et al., 2018).

418 Regardless of fungicide type, honey bees removed less nectar from artificial flowers with copper

419 and propiconazole residues, demonstrating that fungicide application and residual contamination

420 of floral rewards can affect forager decisions. Exposure to fungicide-contaminated rewards can

421 affect pollinator health and pollination in agroecosystems, including almond. Such consequences

422 range from negative effects on larval development, pollinator cognition, and even mortality

423 (Johnson, 2015), as has been observed with honey bee workers directly exposed to a range of

424 fungicides commonly employed for pathogen control in almonds. Although fungicide residues in

425 nectar deterred honey bees, these shifts in foraging behavior did not translate to noticeable

426 effects on pollination services in almonds measured through pollen germination and tube

427 number. Honey bees foraging on almond flowers however often alternate between foraging for

428 nectar or pollen (Bosch and Blas, 1994). These nectar and pollen foragers differ markedly in the

429 quality of services that they provide, with pollen foragers being on average five times more

430 effective in affecting fruit set than those foraging for nectar (Bosch and Blas, 1994). Given that

431 our flowers were exposed for a long enough duration to allow pollinators to forage for both

432 resources, those that foraged for pollen alone likely conferred adequate pollination observed in

433 our experiment. 
Our results highlight multiple orchard management practices that can shape the assembly

435 of crop-associated microbiota during flowering and pollination. We documented temporal

436 changes in microbial abundance and composition, but also detected effects of managed

437 pollinators and natural areas, suggesting a key role of immigration in determining species

438 composition in many floral tissues. Combined with the potential for agrochemicals to

439 differentially affect microbial growth and species interactions, we outline a few factors that

440 likely contribute to flower microbiome assembly. Because flowers form the template for

441 potential reproductive output that is translated through interactions with pollinators,

442 understanding linkages between management, the assembly of the floral microbiome and its

443 impact on pollination in crops can reveal how microbial interactions affect both crop yield and

444 quality.

446 Authors' contributions

447 RS conceived the idea for the study and collaborated with DC, JB, TF, NW, and RV in designing

448 the survey and experiments performed. RS carried out the survey, experiments, analyses, and

449 wrote the first draft of the manuscript. DC and JI contributed ArcGIS data and analysis. All

450 authors contributed to revisions.

\section{Acknowledgements}

453 We are grateful to the growers for permission to work on their properties, and E. Niño for access

454 to an apiary. We also thank E. Bloom, S. Cibotti, L. Hack, G. Hall, W. Menendez, and H. Pathak

455 for field and laboratory assistance. This work was supported by a USDA ELI Postdoctoral

456 Fellowship (2017-67012-26104) awarded to RS, USDA NIFA (grant accession nos 1003539 and 
1014754) to DC, Project Number 6036-2200-028-00-D for JB, and UC Davis start-up and Hatch

NE1501 funds awarded to RV.

\section{References}

Aizenberg-Gershtein, Y., Izhaki, I., and Halpern, M. (2013) Do honeybees shape the bacterial

Allard, S.M. and Micallef, S.A. (2019) The Plant Microbiome: Diversity, Dynamics, and Role in Food Safety. In Safety and Practice for Organic Food. Elsevier, pp. 229-257.

Bates, D., Maechler, M., Bolker, B., and Walker, S. (2014) lme4: Linear mixed-effects models using Eigen and S4. R Package Version 1: 1-23.

Berg, M. and Koskella, B. (2018) Nutrient-and dose-dependent microbiome-mediated protection against a plant pathogen. Curr Biol 28: 2487-2492. e3.

Bosch, J. and Blas, M. (1994) Foraging behaviour and pollinating efficiency of Osmia cornuta and Apis mellifera on almond (Hymenoptera, Megachilidae and Apidae). Appl Entomol Zool 29: 1-9.

Brittain C., Williams N.M., Kremen C., and Klein, AM (2013) Synergistic effects of non-Apis bees and honey bees for pollination services. Proc R Soc B Biol Sci 280: 20122767.

Busby, P.E., Soman, C., Wagner, M.R., Friesen, M.L., Kremer, J., Bennett, A., et al. (2017) Research priorities for harnessing plant microbiomes in sustainable agriculture. PLOS Biol 15: e2001793.

Callahan, B.J., McMurdie P.J., Rosen M.J., Han A.W., Johnson, A.J., and Holmes S.P. (2016) DADA2: high-resolution sample inference from Illumina amplicon data. Nat Methods 13: 581-583.

Edwards, J., Johnson, C., Santos-Medellín, C., Lurie, E., Podishetty, N.K., Bhatnagar, S., et al. (2015) Structure, variation, and assembly of the root-associated microbiomes of rice. Proc Natl Acad Sci 112: E911-E920.

Frazier, M.T., Mullin, C.A., Frazier, J.L., Ashcraft, S.A., Leslie, T.W., Mussen, E.C., and Drummond, F.A. (2015) Assessing honey bee (Hymenoptera: Apidae) foraging populations and the potential impact of pesticides on eight U.S. crops. J Econ Entomol 108: 2141-2152.

Gary, N.E., Witherell, P.C., and Marston, J.M. (1978) Distribution and foraging activities of honeybees during almond pollination. J Apic Res 17: 188-194.

Good, A.P., Gauthier, M.-P.L., Vannette, R.L., and Fukami, T. (2014) Honey bees avoid nectar colonized by three bacterial species, but not by a yeast species, Isolated from the bee gut. PLOS ONE 9: e86494.

Grab, H., Branstetter, M.G., Amon, N., Urban-Mead, K.R., Park, M.G., Gibbs, J., et al. (2019) Agriculturally dominated landscapes reduce bee phylogenetic diversity and pollination services. Science 363: 282-284.

Grady, K.L., Sorensen, J.W., Stopnisek, N., Guittar, J., and Shade, A. (2019) Assembly and seasonality of core phyllosphere microbiota on perennial biofuel crops. Nat Commun 10: $1-10$. 
Graystock, P., Rehan, S.M., and McFrederick, Q.S. (2017) Hunting for healthy microbiomes: determining the core microbiomes of Ceratina, Megalopta, and Apis bees and how they associate with microbes in bee collected pollen. Conserv Genet 18: 701-711.

Herrera, C.M., Pozo, M.I., and Medrano, M. (2013) Yeasts in nectar of an early-blooming herb: sought by bumble bees, detrimental to plant fecundity. Ecology 94: 273-279.

Johnson, R.M. (2015) Honey Bee Toxicology. Annu Rev Entomol 60: 415-434.

Junker, R.R. and Keller, A.(2015) Microhabitat heterogeneity across leaves and flower organs promotes bacterial diversity. FEMS Micro Ecol 91: fiv097.

Kevan, P.G., Kapongo, J., Al-mazra'awi, M., and Shipp, L. (2008) Honey bees, bumble bees and biocontrol. Bee Pollinat Agric Ecosyst Oxf Univ Press $N$ Y.

Klein, A.-M., Brittain, C., Hendrix, S.D., Thorp, R., Williams, N., and Kremen, C. (2012) Wild pollination services to California almond rely on semi-natural habitat. J Appl Ecol

Klein, A.-M., Vaissière, B.E., Cane, J.H., Steffan-Dewenter, I., Cunningham, S.A., Kremen, C., and Tscharntke, T. (2007) Importance of pollinators in changing landscapes for world crops. Proc R Soc B Biol Sci 274: 303-313.

Koch, H., Abrol, D.P., Li, J., and Schmid-Hempel, P. (2013) Diversity and evolutionary patterns of bacterial gut associates of corbiculate bees. Mol Ecol 22: 2028-2044.

Kovács-Hostyánszki, A., Espíndola, A., Vanbergen, A.J., Settele, J., Kremen, C., and Dicks, L.V. (2017) Ecological intensification to mitigate impacts of conventional intensive land use on pollinators and pollination. Ecol Lett 20: 673-689.

Kremen, C., Albrech, M., and Ponisio, L.C. (2019) Restoring pollinator communities and pollination services in hedgerows in intensively-managed agricultural landscapes. In The Ecology of Hedgerows and Field Margins. Routledge, pp. 163-185.

Kremen, C., Williams, N.M., and Thorp, R.W. (2002) Crop pollination from native bees at risk from agricultural intensification. Proc Natl Acad Sci 99: 16812-16816.

Lautenbach, S., Seppelt, R., Liebscher, J., and Dormann, C.F. (2012) Spatial and temporal trends of global pollination benefit. PLOS ONE 7: e35954.

Lindow, S.E. and Andersen, G.L. (1996) Influence of immigration on epiphytic bacterial populations on navel orange leaves. Appl Environ Microbiol 62: 2978-2987.

Lymperopoulou, D.S., Adams, R.I., and Lindow, S.E. (2016) Contribution of vegetation to the microbial composition of nearby outdoor air. Appl Environ Microbiol 82: 3822-3833.

Menzler-Hokkanen, I. and Hokkanen, H.M. (2017) Entomovectoring: An agroecological practice of using bees for biocontrol. Agroecol Pract Sustain Agric Princ Appl Mak Transit 183.

Mueller, U.G. and Sachs, J.L. (2015) Engineering microbiomes to improve plant and animal health. Trends Microbiol 23: 606-617.

Peay, K.G., Belisle, M., and Fukami, T. (2011) Phylogenetic relatedness predicts priority effects in nectar yeast communities. Proc R Soc B Biol Sci 279: 749-758.

Pii, Y., Mimmo, T., Tomasi, N., Terzano, R., Cesco, S., and Crecchio, C. (2015) Microbial interactions in the rhizosphere: beneficial influences of plant growth-promoting rhizobacteria on nutrient acquisition process. A review. Biol Fertil Soils 51: 403-415.

R Core Team (2013) R: A Language and Environment for Statistical Computing, Vienna, Austria: R Foundation for Statistical Computing.

Rering, C.C., Beck, J.J., Hall, G.W., McCartney, M.M., and Vannette, R.L. (2018) Nectarinhabiting microorganisms influence nectar volatile composition and attractiveness to a generalist pollinator. New Phytol 220: 750-759. 

shakers: Bumble bee foraging behavior shapes the dispersal of microbes among and within flowers. Ecosphere 10: e02714.

Schaeffer, R.N. and Irwin, R.E. (2014) Yeasts in nectar enhance male fitness in a montane perennial herb. Ecology 95: 1792-1798.

Schaeffer, R.N., Mei, Y.Z., Andicoechea, J., Manson, J.S., and Irwin, R.E. (2017) Consequences of a nectar yeast for pollinator preference and performance. Funct Ecol 31: 613-621.

Schaeffer, R.N., Rering, C.C., Maalouf, I., Beck, J.J., and Vannette, R.L. (2019) Microbial metabolites elicit distinct olfactory and gustatory preferences in bumblebees. Biol Lett 15: 20190132.

Schaeffer, R.N., Vannette, R.L., Brittain, C., Williams, N.M., and Fukami, T. (2017) Non-target effects of fungicides on nectar-inhabiting fungi of almond flowers. Environ Microbiol Rep 9: 79-84.

Scheper, J., Bommarco, R., Holzschuh, A., Potts, S.G., Riedinger, V., Roberts, S.P.M., et al. (2015) Local and landscape-level floral resources explain effects of wildflower strips on wild bees across four European countries. J Appl Ecol 52: 1165-1175.

Sumner, D.A., Matthews, W.A., Medellín-Azuara, J., and Bradley, A. (2014) The economic impacts of the California almond industry. Rep Prep Almond Board Calif Univ Calif Davis CA.

Thomson, J.D. and Goodell, K. (2001) Pollen removal and deposition by honeybee and bumblebee visitors to apple and almond flowers. J Appl Ecol 38: 1032-1044.

Toju, H., Peay, K.G., Yamamichi, M., Narisawa, K., Hiruma, K., Naito, K., et al. (2018) Core microbiomes for sustainable agroecosystems. Nat Plants 4: 247.

Vannette Rachel L., Gauthier Marie-Pierre L., and Fukami Tadashi (2013) Nectar bacteria, but not yeast, weaken a plant-pollinator mutualism. Proc R Soc B Biol Sci 280: 20122601.

Vannette, R.L. and Fukami, T. (2018) Contrasting effects of yeasts and bacteria on floral nectar traits. Ann Bot 121: 1243-1349.

Vannette, R.L. and Fukami, T. (2017) Dispersal enhances beta diversity in nectar microbes. Ecol Lett 20: 901-910.

Vannette, R.L. and Fukami, T. (2014) Historical contingency in species interactions: towards niche-based predictions. Ecol Lett 17: 115-124.

Vurukonda, S.S.K.P., Vardharajula, S., Shrivastava, M., and SkZ, A. (2016) Enhancement of drought stress tolerance in crops by plant growth promoting rhizobacteria. Microbiol Res 184: $13-24$.

Williams, N.M., Ward, K.L., Pope, N., Isaacs, R., Wilson, J., May, E.A., et al. (2015) Native wildflower plantings support wild bee abundance and diversity in agricultural landscapes across the United States. Ecol Appl 25: 2119-2131.

Yi, W., Law, S.E., Mccoy, D., and Wetzstein, H.Y. (2006) Stigma development and receptivity in almond (Prunus dulcis). Ann Bot 97: 57-63.

584 Figure 1. Map of the study area, showing heterogeneity in land-cover classes (Almond: brown,

585 Forest: green; Shrubland: blue; Grass/Pasture: tan) across the Sacramento Valley of California, a 
major production area for almond. Points represent sampled orchards and are color-coded by management scheme (Conventional: black; Forb-amended: purple; Organic: blue). on YMA (fungi) associated with floral tissues (Anther, Nectary, or Petal) of almond. Flowers were sampled at two stages of bloom (Early or Peak) from orchards that employed different

591 management schemes (Conventional, Forb-amended, or Organic).

592 Figure 3. Observed sequence variant richness of a) bacteria and b) fungi associated with floral

593 tissues (Anther, Nectary, or Petal) of almond flowers. Flowers were sampled at two stages of

594 bloom (Early or Peak) from orchards that employed different management schemes

595 (Conventional, Forb-amended, or Organic).

596 Figure 4. Average relative abundance (Proportion of Sequences) of a) bacterial and b) fungal

597 families associated with floral tissues (Anther, Nectary, or Petal) of almond. Flowers were

598 collected from orchards that vary in management scheme (Conventional, Forb-amended, or

599 Organic).

600 Figure 5. Mean abundance of colony-forming units (CFUs) of bacteria (gold = Asaia, red =

601 Neokamagataea $)$ and yeasts ( $\tan =$ Aureobasidium, blue = Metschnikowia $)$ when introduced in

602 different orders (Bacteria-first, Yeasts-first, or Simultaneously) to nectar environments treated

603 with fungicides (Copper or Propiconazole at $7500 \mathrm{ppb}$ ). Simultaneous introductions were carried

604 out on day 0 , while sequential introductions on days 0 and 2.

605 Figure 6. Nectar remaining $(\mathrm{g})$ in artificial almond flowers treated with fungicides (Copper or

606 Propiconazole) and nectar-inhabiting microbes (red = bacterium Neokogamataea thailandica,

607 blue = yeast Metschnikowia reukaufii). 
bioRxiv preprint doi: https://doi org/10.1101/202011.05.367003; this version posted November 5, 2020. The copyright holder for this preprint

(which was not certified by peer review) is the author/funder, who has granted bioRxiv a license to display the preprint in perpetuity. It is made available under aCC-BY-NC-ND 4.0 International license.

609 Table 1. PERMANOVA results of Bray-Curtis dissimilarity between bacterial and fungal

610 communities associated with almond floral tissues.

611

Table 1 - PERMANOVA results of Bray-Curtis dissimilarity between bacterial and fungal communities associated with almond floral tissues

\begin{tabular}{|c|c|c|c|c|c|c|c|c|c|}
\hline \multirow{2}{*}{$\begin{array}{l}\text { Bacteria } \\
\text { Variable }\end{array}$} & \multicolumn{3}{|c|}{ Anther } & \multicolumn{3}{|c|}{ Nectary } & \multicolumn{3}{|c|}{ Petal } \\
\hline & $R^{2}$ & $F$ & $P$ & $R^{2}$ & $F$ & $P$ & $R^{2}$ & $F$ & $P$ \\
\hline Management & 0.043 & 1.10 & 0.299 & 0.039 & 0.63 & 0.740 & 0.061 & 1.26 & 0.157 \\
\hline Bloom stage & 0.033 & 1.71 & 0.072 & 0.075 & 2.42 & 0.047 & 0.069 & 2.86 & 0.003 \\
\hline Site & 0.024 & 1.23 & 0.234 & 0.030 & 0.96 & 0.374 & 0.018 & 0.75 & 0.744 \\
\hline Management x Bloom stage & 0.022 & 0.56 & 0.963 & 0.027 & 0.87 & 0.471 & 0.062 & 1.30 & 0.147 \\
\hline Management x Site & 0.029 & 0.76 & 0.791 & 0.019 & 0.30 & 0.983 & 0.036 & 0.74 & 0.848 \\
\hline Bloom stage $\mathrm{x}$ Site & 0.013 & 0.67 & 0.797 & - & - & - & 0.026 & 1.10 & 0.360 \\
\hline Management $x$ Bloom stage $\mathrm{x}$ Site & 0.043 & 1.12 & 0.323 & - & - & - & 0.027 & 0.57 & 0.974 \\
\hline Natural habitat & 0.041 & 2.10 & 0.017 & 0.010 & 0.33 & 0.880 & 0.022 & 0.90 & 0.564 \\
\hline Apiary distance & 0.017 & 0.90 & 0.525 & 0.176 & 5.65 & 0.001 & 0.032 & 1.33 & 0.182 \\
\hline Fungi & \multicolumn{3}{|c|}{ Anther } & \multicolumn{3}{|c|}{ Nectary } & \multicolumn{3}{|c|}{ Petal } \\
\hline Variable & $R^{2}$ & $F$ & $P$ & $R^{2}$ & $F$ & $P$ & $R^{2}$ & $F$ & $P$ \\
\hline Management & 0.073 & 2.11 & 0.001 & 0.071 & 1.54 & 0.077 & 0.078 & 2.60 & 0.005 \\
\hline Bloom stage & 0.137 & 7.89 & 0.001 & 0.146 & 6.33 & 0.001 & 0.210 & 14.01 & 0.001 \\
\hline Site & 0.016 & 0.94 & 0.528 & 0.037 & 1.61 & 0.107 & 0.012 & 0.83 & 0.589 \\
\hline Management x Bloom stage & 0.057 & 1.65 & 0.024 & 0.051 & 1.09 & 0.348 & 0.039 & 1.31 & 0.189 \\
\hline Management $x$ Site & 0.032 & 0.94 & 0.570 & 0.067 & 1.46 & 0.101 & 0.019 & 0.64 & 0.883 \\
\hline Bloom stage $\mathrm{x}$ Site & 0.020 & 1.16 & 0.257 & 0.044 & 1.89 & 0.056 & 0.016 & 1.07 & 0.388 \\
\hline Management $x$ Bloom stage $\mathrm{x}$ Site & 0.033 & 0.97 & 0.511 & 0.044 & 0.96 & 0.515 & 0.022 & 0.74 & 0.774 \\
\hline Natural habitat & 0.029 & 1.65 & 0.056 & 0.053 & 2.30 & 0.016 & 0.052 & 3.44 & 0.004 \\
\hline Apiary distance & 0.032 & 1.83 & 0.021 & 0.027 & 1.15 & 0.304 & 0.056 & 3.73 & 0.002 \\
\hline
\end{tabular}

612 


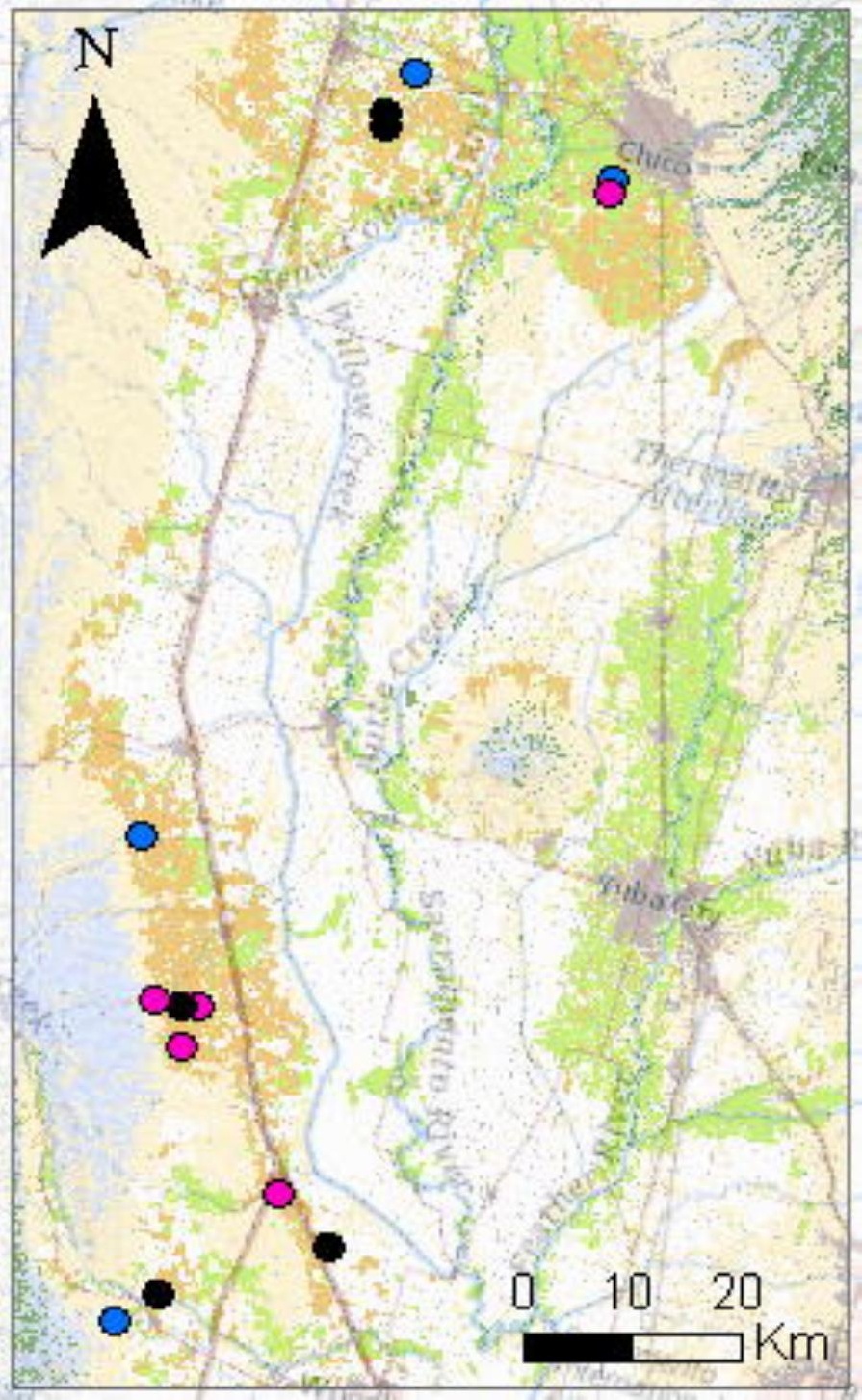







Control

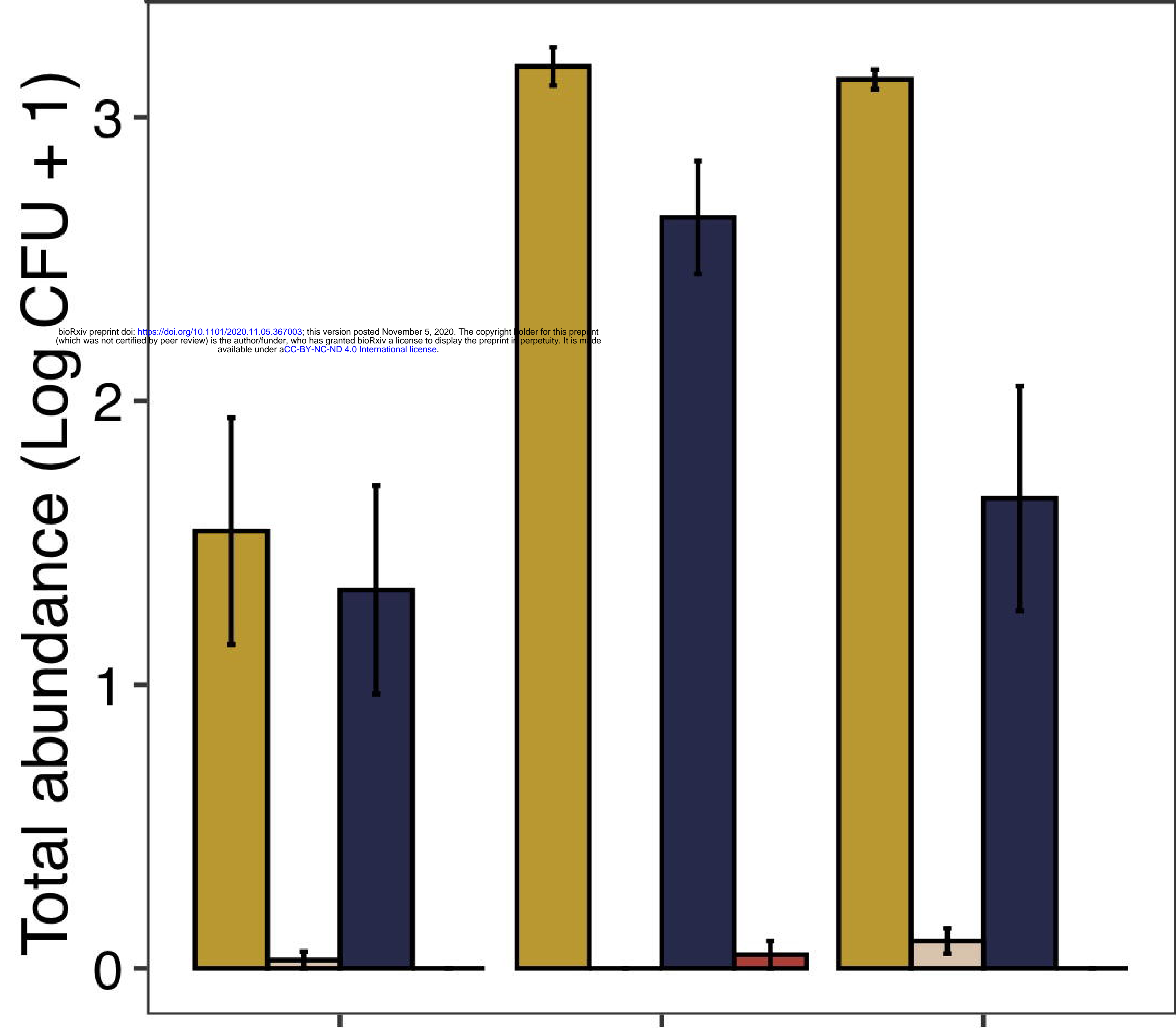

Bacteria-first Yeast-first Simultaneous
Copper

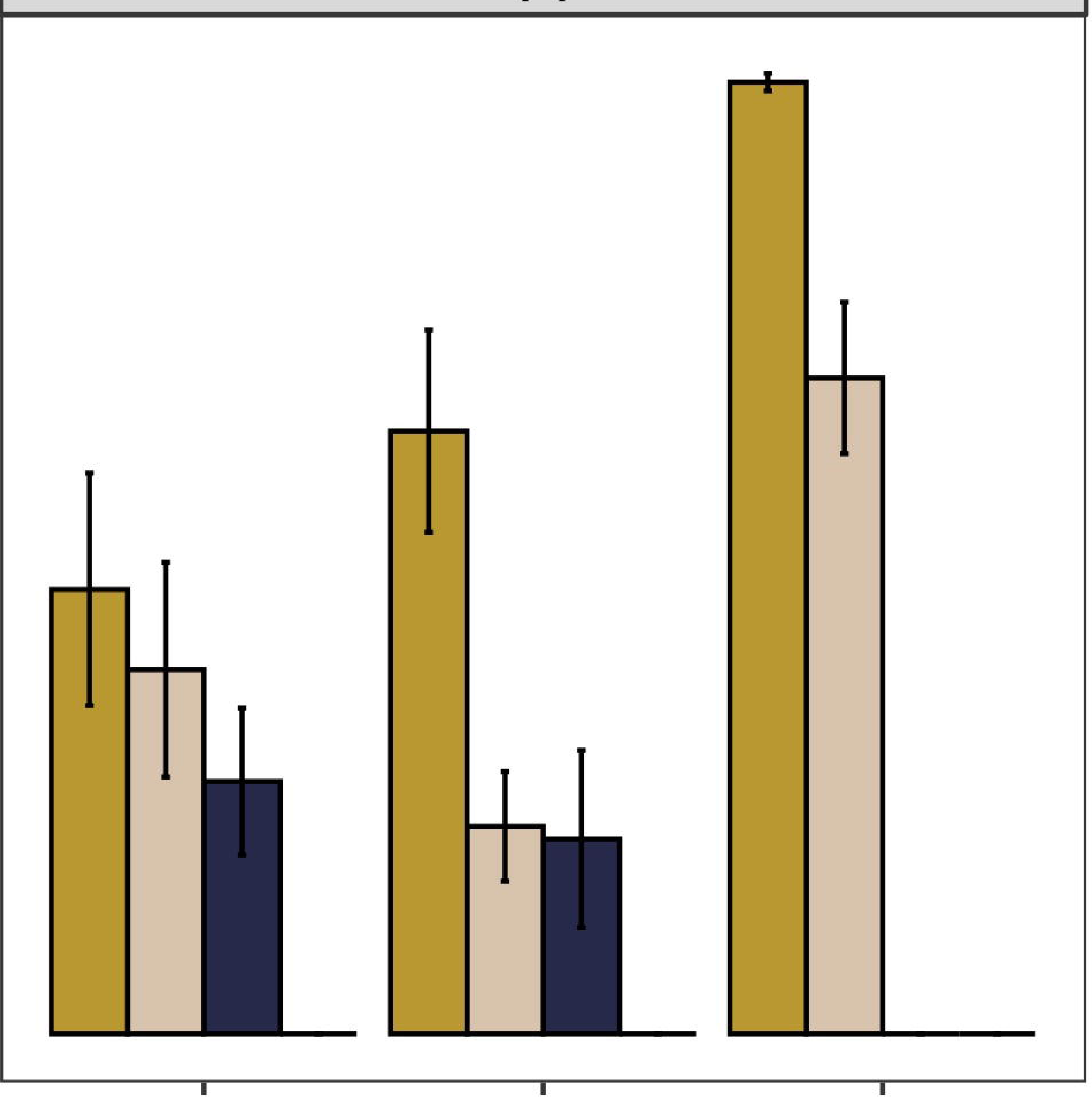

Bacteria-first Yeast-first Simultaneous Inoculation order
Propiconazole

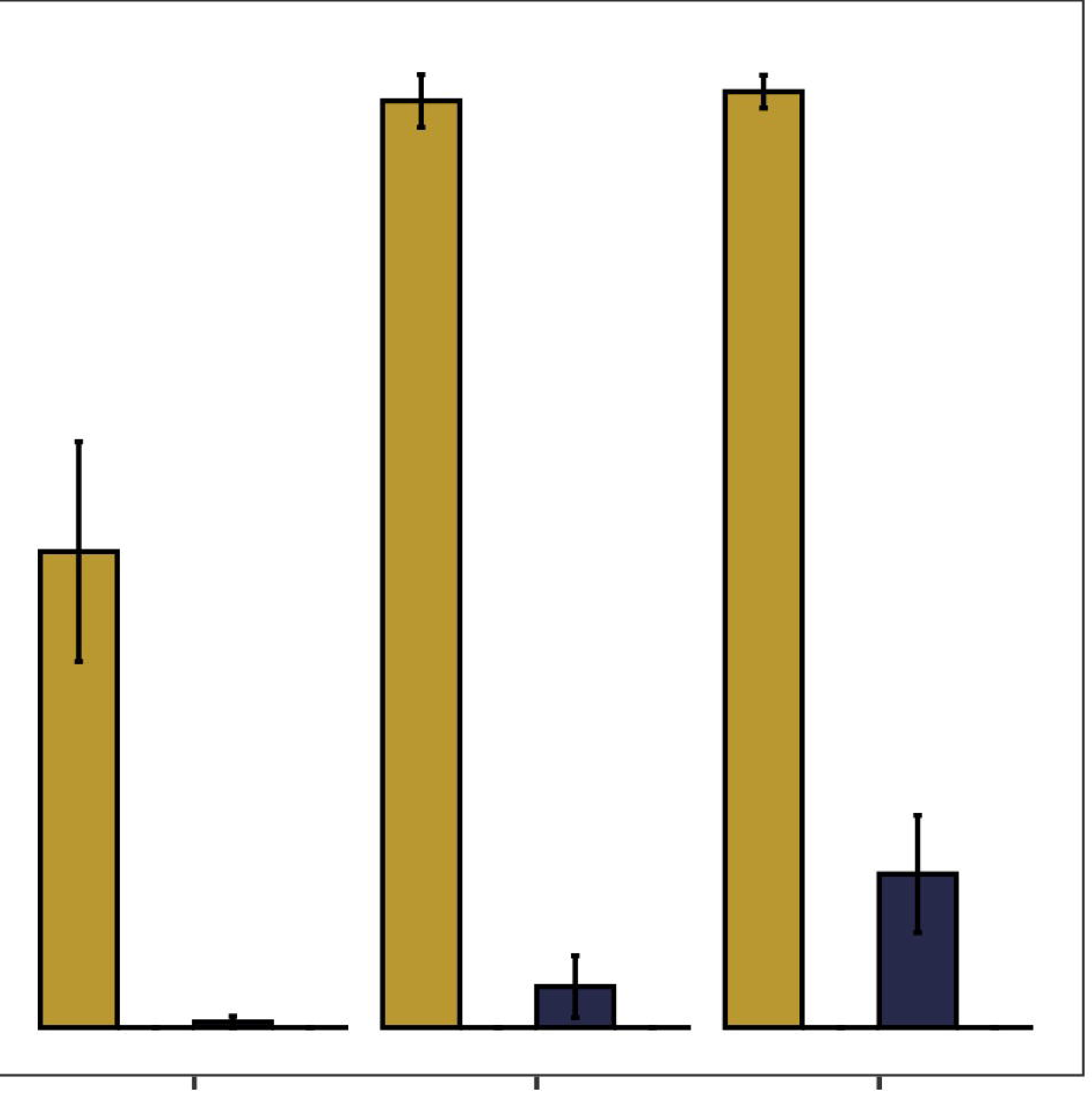

Bacteria-first Yeast-first Simultaneous
Species

$\square$ Asaia

Aureobasidium

Metschnikowia

Neokamagataea 


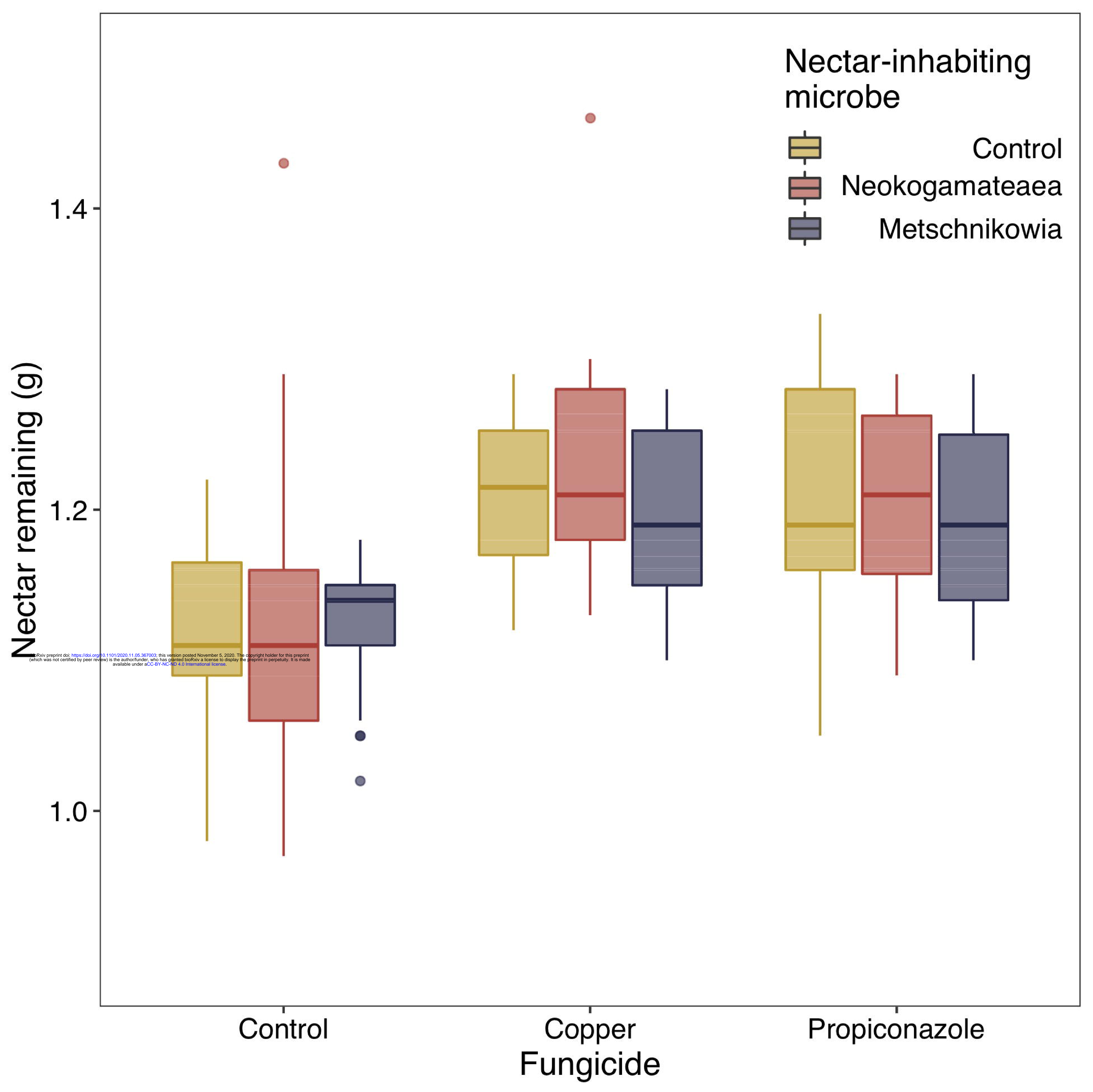

